\title{
Radio Pulse Propagation by a Reflection Process at the Lower Ionosphere
}

\author{
J. Ralph Johler \\ Contribution from Central Radio Propagation Laboratory, National Bureau of \\ Standards, Boulder, Colo.
}

(Received May 3, 1963)

\begin{abstract}
The groundwave pulse has been considered in detail by Johler [1962, 1963b]. A theory was presented as a direct numerical and analytic evaluation of the Fourier transform integral. Such a theory can be extended to treat pulsed ionospheric waves.

The reflection process for various model anistoropic ionospheres has been studied in detail in previous papers by Johler [1962]. The analysis of the reflection coefficient thus demonstrated is employed to construct transfer functions for the ionospheric wave. These transfer functions are then introduced into the Fourier transform of the pulse, and the behavior of LF pulses propagated by a reflection process at the ionosphere is determined for three models for the lower ionosphere. The composite ground and ionospheric pulse is formed, and a method of tagging a point-in-time on the pulse is detailed.
\end{abstract}

\section{Introduction}

The low frequency radio signal [Johler, 1962] propagated from a transmitter at the surface of the terrestrial sphere can overcome the curvature of the earth and reach a receiver some distance away on the surface of the earth by diffraction as a groundwave pulse or by a single reflection or multiple reflections at the lower ionosphere. Numerous investigators have treated the groundwave pulse or signal theoretically [Novikov, 1960; Johler and Walters, 1959; Keilson and Row, 1959; Pekeris and Alterman, 1957; Wait, 1955 and 1959]. Thus, for example, Wait and Froese [1955]; Wait and Conda [1959] have developed closed form solutions for certain pulses for relatively simple situations. On the other hand, very little has been accomplished on the pulse signal reflected at the ionosphere at LF. A procedure was outlined by Johler [1962] for treating theoretically both the groundwave and the ionospheric wave pulses by a direct numerical integration of the Fourier transform-integral. Thus, regarding the continuous time-harmonic-wave solution of Maxwell's equations, $\frac{\partial}{\partial t} \vec{E}=i \omega \vec{E} ; \frac{\partial}{\partial t} \vec{H}=i \omega \vec{H}$, as the transform or transfer function of the propagation medium, it is no longer necessary for the case of linear amplitude transforms to resolve Maxwell's equations for each pulse. It is only necessary to form the product of the pulse transform and the complex transfer function of the medium and integrate to the time domain.

The reflection process for various model anisotropic ionospheres has been studied in considerable detail by Johler [1962, 1963a; Johler and Harper, 1962a and 1962b]. Results obtained with the aid of this analysis are employed in this paper to construct three transfer functions for three models of the propagation medium. These transforms are then introduced into the integrand of the Fourier integral of specified pulses, and the behavior of such pulses propagated by reflection at the lower ionosphere is demonstrated. The composite ground and ionospheric pulse is formed, and the methods for tagging a point-in-time [Johler, 1963b] on the pulse are detailed.

1 This work was sponsored by the Advanced Research Projects Agency under ARPA Order No. 183-62, Amendment 4, or NBS Project 85411initiated under this contract. This work forms the theoretical basis for the experimental program pursuant under the same contract on NBS Project 85461 . 


\section{Theory of Transient Propagation}

A radio signal, $\vec{E}(t, d), \mathrm{v} / \mathrm{m}$ or $\vec{H}(t, d)$ amp-turns $/ \mathrm{m}$, at a time, $t$, and a distance, $d$, along the surface of the terrestrial sphere relative to a transmitter is related to the continuous time harmonic solution of Maxwell's equations, $\vec{E}(\omega, d)$ or $\vec{H}(\omega, d)$ at a frequency $f=\omega / 2 \pi$ c/s by the Fourier transform-integral for linear amplitude restrictions,

$$
E\left(t^{\prime}, d\right)=\frac{1}{2 \pi} \int_{-\infty}^{\infty} \exp \left(i \omega t^{\prime}\right) E(\omega, d) f_{r}(\omega) \int_{0}^{\infty} \exp (-i \omega t) F_{s}(t) d t d \omega
$$

where $f_{r}(\omega)$ is the transfer function of the receiver or measuring device and $F_{s}(t)$ is the transient dipole source current moment, and the local time, $t^{\prime}$, is defined,

$$
t^{\prime}=t-\eta_{1} d / c,
$$

where $\eta_{1}$ is the index of refraction of air $\left(\eta_{1} \sim 1.000338\right.$ at the surface of the earth), and $c$ is the speed of light $\left(c \sim 2.997925\left(10^{8}\right) \mathrm{m} / \mathrm{sec}\right)$. The propagation medium transfer function, $E(\omega, d)$, can be split geometrically-optically [Johler, 1962] into the sum of a multiplicity of ordered rays, $j=0,1,2,3 \ldots$.

$$
E(\omega, d)=\sum_{j=0}^{P} E_{j}(\omega, d)
$$

where $j=0$ corresponds to the ground wave, $j=1$ corresponds to the first ionospheric reflection, etc. As a consequence of these rays, the pulse fields exhibit a corresponding series,

$$
E\left(t^{\prime}, d\right)=\sum_{j=0}^{P} E_{j}\left(t^{\prime}, d\right)
$$

separated in time by ever later times, $t^{\prime}$, as the order, $j$, is increased. The groundwave, $j=0$, and the first ionospheric reflection will be considered in this paper. Obviously the latter $(j=1)$ will arrive at the receiver later, since the ray length between the transmitter, the ionosphere, and the receiver is longer than the distance, $d$, along the surface of the earth, to the receiver appropriate to the groundwave, $j=0$. These facts have been verified experimentally [Johler, 1962], and the ray theory for propagation between two points, $s$ (source), and $o$ (observer) on the surface of the earth can be summarized as shown diagrammatically, figure 1.

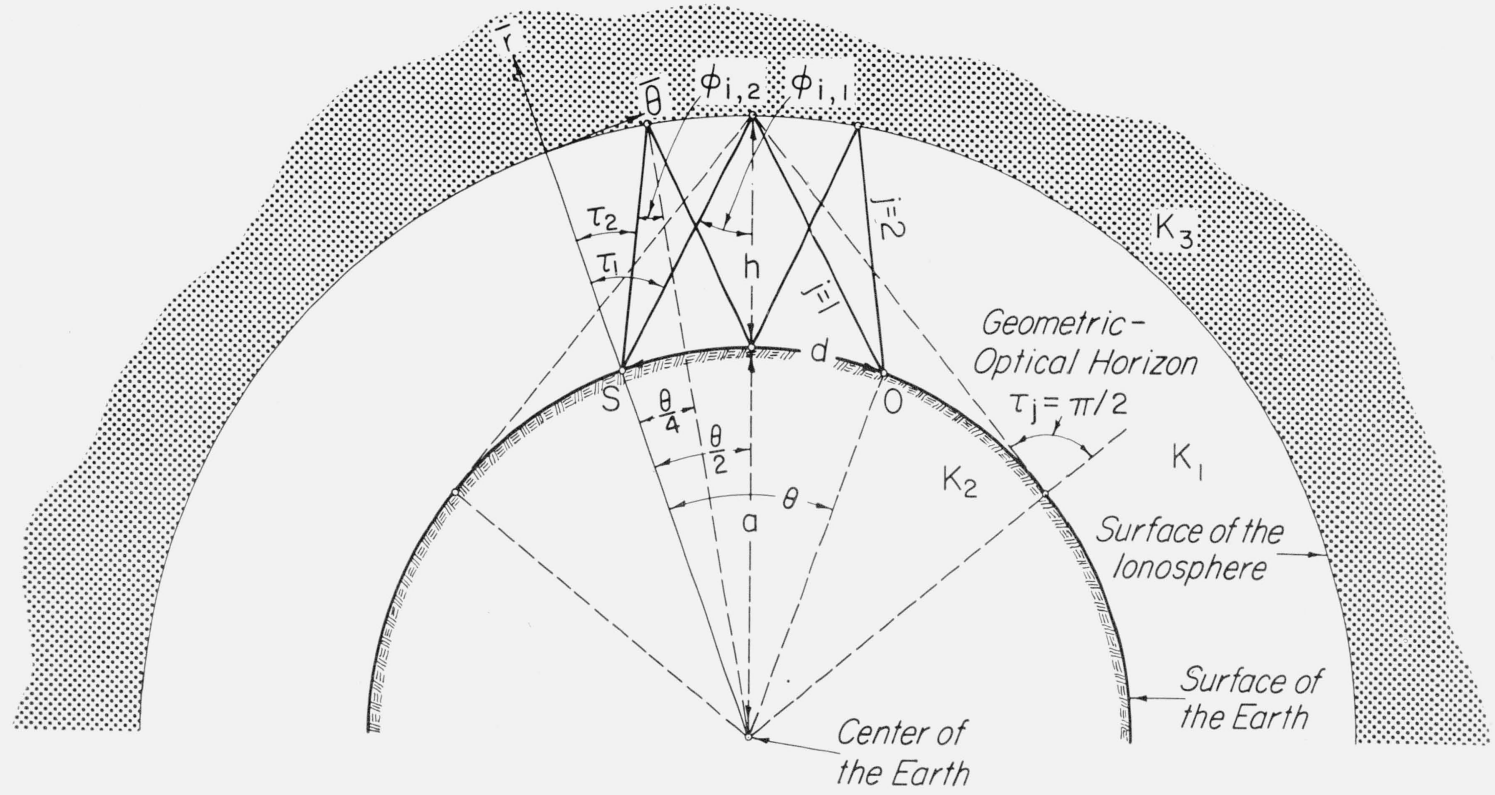

Figure 1. Propagation media and boundaries, illustrating various geometric-optical rays in the vicinity of the terrestrial sphere. 
The electrical properties of the three principal media of propagation are described by the wave numbers, $k_{1}, k_{2}, k_{3}$,

$$
\begin{gathered}
k_{1}=\frac{\omega}{c} \eta_{1} \\
k_{2}=\frac{\omega}{c} \sqrt{\epsilon_{2}-i \frac{\sigma \mu_{0} c^{2}}{\omega},} \\
k_{3}=\frac{\omega}{c} \eta_{0, e}^{i, r}
\end{gathered}
$$

where $\epsilon_{2}$ is the dielectric constant relative to a vacuum, $\epsilon_{2} \epsilon_{0}=\epsilon$ the permittivity, $\epsilon_{0}=\frac{1}{c^{2} \mu_{0}}$, and $\mu_{0}=4 \pi\left(10^{-7}\right) \mathrm{h} / \mathrm{m}$, the permeability of space; $\sigma$, mhos/meters is the conductivity of the ground. The index of refraction for the ionosphere, $\eta_{0, e}^{i, r}$, is quite complicated, and there are four such indices for a homogeneous layer, ordinary $(o)$ and extraordinary $(e)$, upgoing $(i)$ and ordinary $(o)$ and extraordinary $(e)$, downgoing $(r)$ indexes. Furthermore, the electrical properties of $k_{3}$ vary with altitude, $h, k_{3}=k_{3}(h)$ which fact is taken into account in the reflection coefficient, $T$, of the ionosphere. The lowest electron density considered is taken as the lower boundary at the altitude, $h$, for purposes of geometric-optical rays, figure 1.

The groundwave $(j=0)$ signal, $E_{0}\left(t^{\prime}, d\right)$ is considered to be the pulse which travels from $s$ to 0 , figure 1, along the surface of the earth taking into account the electrical properties of the earth, $k_{2}$, and the medium, $k_{1}$, assumed to be of infinite extent. This is the earliest signal to arrive at $o$, and the earliest time such a signal can arrive at $o$ is $t^{\prime}=0$ or $t=\eta_{1} d / c$. Somewhat later (about 20 to $40 \mu \mathrm{sec}$ ) the signal propagated over the ray, $j=1$, and reflected from the lower ionosphere at a height, $h$, and angle of incidence, $\phi_{i, 1}$, will arrive at a local time, $t_{j}^{\prime}=t_{1}^{\prime}>0$, or will take on appreciable amplitude at a time, $t_{1}^{\prime}>0$. Obviously, higher order rays $(j=2$, 3, 4 ...) are possible and indeed have been observed [Johler, 1962]. However, for purposes of illustrating technique the distances, $d=800$ to 2000 statute miles or $\{1200$ to $3000 \mathrm{~km}$ will be considered in which for the assumed models, $j=0$ and $j=1$, are of prime consideration. Indeed, a model will be considered in which the $j=1$ ray, figure 1 , is diffracted beyond the geometrical-optical horizon, or beyond the point at which the ray is grazing incidence on the earth, $\tau_{j}=\tau_{1}=\frac{\pi}{2}$. The rays reflecting to and fro between the earth and the ionosphere are influenced by the local reflection coefficient at the ground surface, $r=a$, and at the ionosphere surface, $r=a+h$. Distance, $d=a \theta$, increases in the positive $\theta$-direction or the signals are assumed to be progressing toward the receiver or observer, $o$, in the positive $\theta$-direction, figure 1.

The transform for the propagation medium, considering the groundwave, $E_{0}(\omega, d)$, and the first ionospheric wave, $E_{1}(\omega, d)$ can be written [Johler, 1962] for vertical dipoles at $s$ and $o$ [Johler, 1961].

$$
E(\omega, d)==E_{0}(\omega, d)+E_{1}(\omega, d)
$$

$$
\begin{aligned}
& E(\omega, d)=2 i \omega C\left[2 \pi \alpha^{2 / 3}\left(k_{1} a\right)^{1 / 3} \frac{d}{a}\right]^{1 / 2}\left\{\frac{\exp \left\{-i\left[\left(k_{1} a\right)^{1 / 3} \tau_{s} \alpha^{2 / 3} \frac{d}{a}+\frac{\alpha d}{2 a}+\frac{\pi}{4}\right]\right\}}{\left[2 \tau_{s}-1 / \delta_{e}^{2}\right]}\right. \\
& \left.+i \omega d D_{1}^{-1} C \exp \left[i \omega\left(t_{1}^{\prime}-t^{\prime}\right)\right] G_{1}^{2} \alpha_{1} F_{1}^{2} T_{e e}\right\}
\end{aligned}
$$

where

and

$$
\begin{gathered}
C=\left(10^{-7}\right) I_{0} l / d \\
G \sim \sin \tau_{1}, \\
t^{\prime}=t-\eta_{1} D_{1} / c, \\
D_{1}=2\left[(a+h) \cos \phi_{i}-a \cos \tau_{1}\right], \\
\phi_{i}=\phi_{i, 1},
\end{gathered}
$$


$\alpha \sim 0.85$, the earth radius factor, $a$ is the radius of the earth, $\tau_{s}$ are the special roots of Riccati's differential equation [Johler, Walters, and Lilley, 1959],

where for vertical polarization $\delta=\delta_{e}$

$$
\frac{d \delta}{d \tau}-2 \delta^{2} \tau+1=0
$$

$$
\delta_{e}=\frac{-i k_{2}^{2} \alpha^{1 / 3} / k_{1}^{2}}{\left(k_{1} a\right)^{1 / 3}\left[\frac{k_{2}^{2}}{k_{1}^{2}-1}\right]^{1 / 2}}
$$

where $k_{1}$ and $k_{2}$ are defined in (5) and (6),

$$
\begin{gathered}
\sin \phi_{i}=\frac{a \sin \frac{\theta}{2}}{\left[2 a(a+h)\left(1-\cos \frac{\theta}{2}\right)+h^{2}\right]^{1 / 2}} \\
\alpha_{1}=\left(1+\frac{h}{a}\right)\left[2 \sin \frac{\theta}{2} / \sin \theta\right]^{1 / 2}\left\{\left[a\left(1-\cos \frac{\theta}{2}\right)+h\right] /\left[(a+h) \cos \frac{\theta}{2}-a\right]\right\}^{1 / 2} A_{1} \\
A_{1} \sim\left(\frac{\pi}{2} z_{1}\right)^{1 / 2} H_{1 / 3}^{(2)}\left(z_{1}\right) \exp \left\{-i\left[5 \pi / 12-z_{1}\right]\right\} \\
z_{1}=\left(k_{1} a \cos ^{3} \tau_{1}\right) /\left(3 \sin ^{2} \tau_{1}\right) \\
F_{1} \cong \pi^{-1 / 2} \exp \left[-i k_{1} a \theta^{\prime}\right] \int_{\infty}^{\infty} \exp [-i 2 \pi / 3] \\
\frac{\exp \left[-i\left(k_{1} a / 2\right)^{1 / 3} \theta^{\prime} \rho\right]}{W_{1}^{\prime}(\rho)-q W_{1}(\rho)}
\end{gathered}
$$

where $\theta^{\prime}=\left(d-d_{H}\right) / a, d_{H}$ is the distance to geometric-optical horizon, figure $1, \tau_{1}=\frac{\pi}{2}$;

$$
q=-i\left(\frac{k_{1} a}{2}\right)^{1 / 3} \frac{k_{1}}{k_{2}} \sqrt{1-\frac{k_{1}^{2}}{k_{2}^{2}}}
$$

[Wait and Conda, 1958; Johler, 1962]. $W_{1}(z)$ and $W_{1}^{\prime}(z)$ are airy integrals related to the Hankel functions, $H_{1 / 3}^{(2)}(z), H_{2 / 3}^{(2)}(z)$,

where

$$
\begin{gathered}
W_{1}(\rho)=\exp [-2 \pi i / 3] \sqrt{\pi / 3}(-\rho)^{1 / 2} H_{1 / 3}^{(2)}\left[\frac{2}{3}(-\rho)^{3 / 2}\right], \\
W_{1}^{\prime}(\rho)=\exp [-4 \pi i / 3] \sqrt{\pi / 3} \rho H_{2 / 3}^{(2)}\left[\frac{2}{3}(-\rho)^{3 / 2}\right],
\end{gathered}
$$

$$
-\frac{\pi}{3} \leqq \arg \rho<\frac{5 \pi}{3}
$$

and $\arg (-\rho)=\arg \rho-\pi, \arg \rho^{m / n}=\frac{m}{n} \arg \rho$ where $m$ and $n$ are integers. $\quad F_{1}$ can also be calculated in the geometric-optical diffraction zone $\left(\tau_{1}=\frac{\pi}{2}\right.$, fig. 1) with the aid of [Wait and Conda, 1958], an alternate formula,

$$
F_{1} \simeq-2 i \sqrt{\pi} \exp \left[-i k_{1} a \theta^{\prime}\right] \sum_{s=0}^{\infty} \frac{\exp \left[-i\left(k_{1} a\right)^{1 / 3} \theta^{\prime} \tau_{s}\right]}{\left(2^{1 / 3} \tau_{s}-q^{2}\right) W_{1}\left(2^{1 / 3} \tau_{s}\right)}
$$

This formula clearly shows the groundwave nature of the diffracted wave, since it is the classical residue series by analogy to first term of (8) and (9), which is the groundwave. $H_{1 / 3}^{(2)}(z)$ was computed with the aid of a computer technique [Berry, 1962] using the relations,

$$
\begin{gathered}
H_{1 / 3}^{(2)}(z)=\left\{-\exp \left(\frac{\pi i}{3}\right) J_{1 / 3}(z)+J_{-1 / 3}(z)\right\} / i \sin \left(-\frac{\pi}{3}\right) \\
H_{2 / 3}^{(2)}(z)=\left\{-\exp \left(\frac{2 \pi i}{3}\right) J_{2 / 3}(z)+J_{-2 / 3}(z)\right\} / i \sin \left(-\frac{2 \pi}{3}\right)
\end{gathered}
$$


where

$$
\begin{aligned}
J_{v}(z)= & \sum_{m=0}^{\infty} \frac{(-1)^{m}(z / 2)^{v+2 m}}{m ! T^{\prime}(v+m+1)} \\
& =\frac{(z / 2)^{v}}{T^{\prime}(v+1)}\left[1-\frac{(z / 2)^{1 / 2}}{1 !(v+1)}+\frac{(z / 2)^{4}}{2 !(v+1)(v+2)}-\frac{(z / 2)^{6}}{3 !(v+1)(v+2)(v+3)}+\ldots\right]\left(v=\frac{1}{3}, \frac{2}{3}\right) .
\end{aligned}
$$

$J_{v}(z)$ is made single valued by specifying $\arg (z / 2)^{v}=v \arg (z / 2)$ for $\Delta-2 \tau<\arg (z / 2) \leq \Delta$. Ordinarily, $\Delta=\pi$ but may have different values, depending upon applications.

As $|z|$ gets large, (27) converges slowly and loses precision. Also, the precision lost in (25) and (26) is serious, since in half of the $z$-plane the terms on the right are nearly negatives of each other when $\operatorname{Im}(z)$ is large. It is therefore necessary to use the asymptotic series,

where,

$$
H_{v}^{(2)}(z)=\frac{\exp \left[-i z+i\left(v+\frac{1}{2}\right) \pi / 2\right]}{\sqrt{\pi z / 2}} T_{v}(2 i z),(-2 \pi<\arg z<\pi)
$$

$$
T_{v}(z)=1+\sum_{n=1}^{\infty} \frac{\left(4 v^{2}-1^{2}\right)\left(4 v^{2}-3^{2}\right) \ldots\left[4 v^{2}-(2 n-1)^{2}\right]}{n !(4 z)^{n}} .
$$

$T_{v}(z)$ is asymptotic except for $v=n+\frac{1}{2}$ and integral $n$, and hence should be terminated at the smallest term, the next term being greater than the error of such a termination.

The reflection coefficient, $T_{e e}$, of the ionosphere comprises a quite complicated computation which has been detailed by Johler [1962] and Johler and Harper [1962a and 1962b]. The ionosphere is considered locally, figure 1, at the reflection point of the ray; that is, a point below which the electron density $<\sim 5-10$ electrons $/ \mathrm{cm}^{3}$. This point in the ionosphere can be tested for contribution to the reflection coefficient by varying the lower boundary. At LF and VLF the reflection coefficient will be found to be determined by greater values of electron density. A plane wave, $E_{t}$, is assumed to be transmitted into the plasma,

where

$$
E_{t}=|\vec{E}| \exp \left[i\left(\omega t-\frac{\omega}{c} \eta D\right)\right]
$$

$$
\eta D=x \sin \phi_{i} \sin \phi_{a}+y \sin \phi_{i} \cos \phi_{a}+z \zeta
$$

in which $\zeta$ is in general a complex number related to the index of refraction, $\eta$,

$$
\eta^{2}=\zeta^{2}+\sin ^{2} \phi_{i}
$$

and a right-handed $x, y, z$ Cartesian coordinate system is assumed locally at the lowermost ionosphere boundary, so that the $y-z$ plane is oriented toward magnetic north and, hence, contains the earth's magnetic field vector, $\overrightarrow{\mathcal{H}}$. The angle $\phi_{a}$, is measured clockwise toward the $x-z$ plane from magnetic north as the orientation of the plane of incidence of the incident wave, and the dip or inclination angle, $I$, is measured downward from the horizontal $x-y$ plane. The angle of incidence, $\phi_{i}$, is measured from the vertical.

The quantity, $\zeta$, is determined by a simultaneous solution of the continuous time-harmonic form of Maxwell's equation,

$$
\frac{\partial}{\partial t} \vec{E}=i \omega \vec{E}, \frac{\partial}{\partial t} \vec{H}=i \omega \vec{H}, \quad \nabla \times \vec{E}+\mu_{0} i \omega \vec{H}=0, \quad \nabla \times \vec{H}-\vec{J}-\epsilon_{0} i \omega \vec{E}=0
$$

and the equation of motion of an electron,

$$
i \omega m \vec{V}+m g \vec{V}+\mu_{0} e(\vec{V} \times \overrightarrow{\mathcal{H}})+e \vec{E}=0,
$$


and $\vec{J}=-N e \vec{V}$ for $N$ electrons/cubic meter of charge, $e$, and mass, $m . \quad \vec{V}$ is the electron velocity vector.

Equations (32) and (33) imply that Maxwell's equations can be written in terms of the dielectric tensor, $\| \epsilon^{\|} \mid$,

$$
\begin{aligned}
& \nabla \times \vec{E}+\mu_{0} i \omega \vec{H}=0 \\
& \nabla \times \vec{H}-\|\epsilon\| \cdot \vec{E}=0
\end{aligned}
$$

where in Cartesian $x, y, z$ coordinates, Johler and Harper [1962b]

where

$$
\|\boldsymbol{\epsilon}\|_{\|}=\epsilon_{0} i \omega\left[\begin{array}{lrr}
1+\frac{s}{s^{2}-h^{2}} & \frac{-i h_{L}}{s^{2}-h^{2}} & \frac{i h_{T}}{s^{2}-h^{2}} \\
\frac{i h_{L}}{s^{2}-h^{2}} & 1-\frac{\left(h_{T}^{2}-s^{2}\right)}{s\left(s^{2}-h^{2}\right)} & \frac{s^{2}-h_{L}^{2}}{s\left(s^{2}-h^{2}\right)} \\
\frac{-i h_{T}}{s^{2}-h^{2}} & \frac{h_{L} h_{T}}{s\left(s^{2}-h^{2}\right)} & 1+\frac{s^{2}-h_{L}^{2}}{s\left(s^{2}-h^{2}\right)}
\end{array}\right]
$$

$$
s=\frac{\omega^{2}}{\omega_{N}^{2}}\left[1-i \frac{\nu}{\omega}\right], \quad h=\frac{\omega_{H} \omega}{\omega_{N}^{2}},
$$

$h_{L}=-h \sin I, h_{T}=h \cos I, \omega_{H}=\mu_{0} e \mathcal{H}|m, \mathcal{H}=| \overrightarrow{\mathcal{H}} \mid$, and $\omega_{N}^{2}=N e^{2} / \epsilon_{0} m$, and $f_{H}=\omega_{H} / 2 \pi$, and $f_{N}=\omega_{N} / 2 \pi$ are the gyro and plasma frequencies, respectively. $\mathcal{H}=H_{m} \cong 0.5$ gauss or $40 \mathrm{amp}-$ turns $/ \mathrm{m}$.

The complex, frequency-dependent parameter, $g$, in the equation of motion (33) is found by an integration over a Maxwellian energy distribution expressed in terms of energy, $e u=\frac{1}{2} m V^{2}$, or $f_{0}=[e / \pi k T]^{3 / 2} \exp [-e u / k T]$, in which there exists momentum transfer collisions with gas molecules proportional to the energy, $u, \nu=\nu(u)$. Here $k T$ is Boltzmann's constant-temperature product. Such an integration can be readily accomplished for a continuous electromagnetic wave of angular frequency, $\omega=2 \pi f$. Thus,

$$
g=\left[-\frac{4 \pi}{3} \int_{0}^{\infty} \frac{u^{3 / 2}}{[\nu(u)+i \Omega]} \frac{\partial}{\partial u}\left\{\left(\frac{e}{\pi k T}\right)^{3 / 2} \exp (-e u / k T) d u\right\}\right]^{-1}-i \Omega
$$

where $\Omega$ is an angular frequency yet to be identified [Johler, 1962 ; Johler and Harper, 1962a and 1962b].

The dielectric tensor can therefore be generalized to take account of collisions, $\nu=\nu(u)$, proportional to energy [Johler and Harper, 1962b,

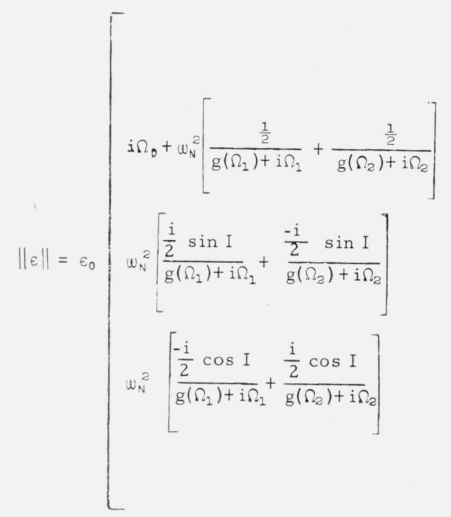

$$
\begin{aligned}
& \omega_{N}^{2}\left[\frac{\frac{i}{2} \sin I}{g\left(\Omega_{1}\right)+i \Omega_{1}}+\frac{\frac{-i}{2} \sin I}{g\left(\Omega_{2}\right)+i \Omega_{2}}\right] \\
& i \Omega_{0}+\omega_{N}^{2}\left[\frac{\cos ^{2} I}{g\left(\Omega_{0}\right)+i \Omega_{0}}+\frac{\frac{1}{2} \sin ^{2} I}{\left.g\left(\Omega_{1}\right)+i \Omega_{1}\right)}+\frac{\frac{1}{2} \sin ^{2} I}{g\left(\Omega_{2}\right)+i \Omega_{2}}\right] \\
& \omega_{N}^{2}\left[\frac{-\sin I \cos I}{g\left(\Omega_{0}\right)+i \Omega_{0}}+\frac{\frac{1}{2} \sin I \cos I}{g\left(\Omega_{1}\right)+i \Omega_{1}}+\frac{\frac{1}{2} \sin I \cos I}{g\left(\Omega_{2}\right)+i \Omega_{2}}\right]
\end{aligned}
$$$$
w_{N}^{2}\left[\frac{\frac{i}{2} \cos I}{g\left(\Omega_{1}\right)+\Omega_{1}}+\frac{\frac{-i}{2} \cos I}{g\left(\Omega_{2}\right)+i \Omega_{1}}\right]
$$$$
\omega_{N}^{2}\left[\frac{-\sin I \cos I}{g\left(\Omega_{0}\right)+i \Omega_{0}}+\frac{\frac{1}{2} \sin I \cos I}{g\left(\Omega_{1}\right)+i \Omega_{1}}+\frac{\frac{1}{2} \sin I \cos I}{g\left(\Omega_{2}\right)+i \Omega_{2}}\right]
$$$$
i \Omega_{0}+w_{N}^{2}\left[\frac{\sin ^{2} I}{g\left(\Omega_{0}\right)+i \Omega_{0}}+\frac{\frac{1}{2} \cos ^{2} I}{g\left(\Omega_{1}\right)+i \Omega_{1}}+\frac{\frac{1}{2} \cos ^{2} I}{g\left(\Omega_{2}\right)+i \Omega_{2}}\right]
$$

where three characteristic frequencies, $\Omega$, have been identified, $\Omega_{0}=\omega, \Omega_{1}=\omega+\omega_{H}$, and $\Omega_{2}=\omega-\omega_{H}$. The vectors, $\vec{V}$ and $\vec{H}$, can be eliminated, whereupon it can be concluded a quartic equation 
in $\zeta$ is satisfied if the field, $\vec{E}$, exists,

$$
a_{4} \zeta^{4}+a_{3} \zeta^{3}+a_{2} \zeta^{2}+a_{1} \zeta+a_{0}=0
$$

Equation (38) is analogous to the Booker [1939] quartic in classical magneto-ionic theory and can be readily solved by various numerical techniques [Johler and Harper, 1962a and $1962 \mathrm{~b}]$. Since the quartic has four roots, the four indexes of refraction of the ionosphere, $\eta_{0, e}^{i, r}$, can be identified with the aid of (31).

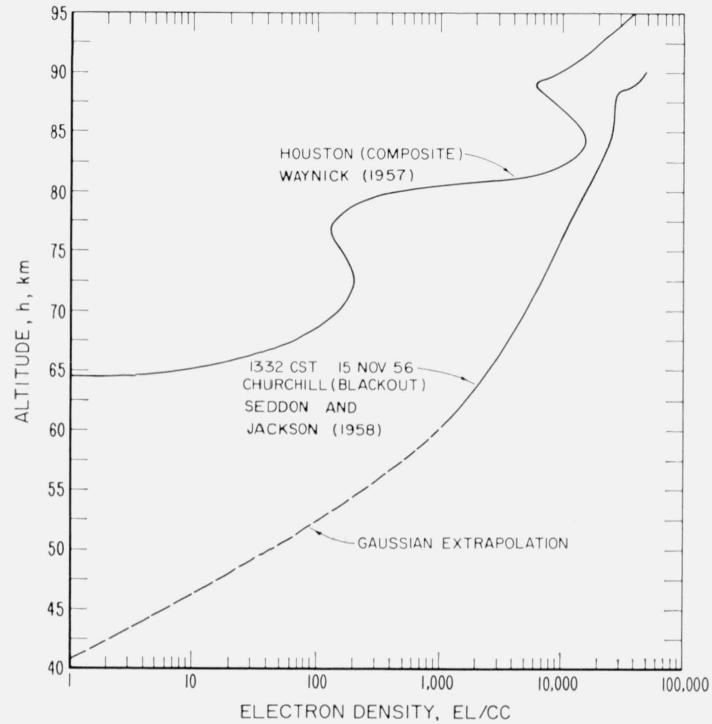

Figure 2. Model ionosphere profiles, N(h), illustrating the variation of the electron density (electrons $/ \mathrm{cm}^{3}$ ) of the lower ionosphere with altitude: (a) Houston Composite model appropriate to lower geographic latitudes; (b) Blackout Model appropriate to higher latitudes (with Gaussian distribution in the region $40-60 \mathrm{~km}$ ).

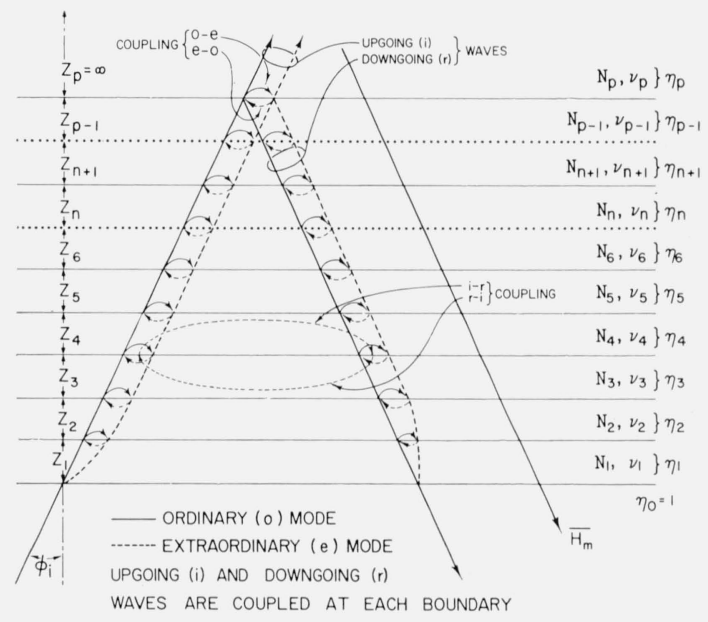

Figure 3. Structure of the stratification employed in the analysis procedure, illustrating the concept of continuous stratification.

The ordinary and extraordinary upgoing and downgoing propagation components are coupled at each boundary.

The detailed structure of two model ionospheres is illustrated, figure 2, as the electron density-altitude, $N(h)$, profile. The collision frequency-altitude, $\nu(h)$ profile has been previously described as the Nicolet/3 profile, Johler [1962]. These structures were represented by a flexible plasma stack of slabs illustrated, figure 3 of arbitrary thickness (except for the topmost slab of thickness, $\left.z_{p}=\infty\right)$. Both the number of such slabs, $p$, and the thickness were quite flexible, since the idea of this analysis is that the measured electron density-altitude and collision frequency-altitude $(N(z)$ and $\nu(z)$, respectively) profiles can be approximated to any desired accuracy by decreasing $z_{n}$ and increasing $p$ simultaneously until a stable reflection process is obtained.

A constant electron density, collision frequency and static magnetic field with respect to altitude, $z$, is of course assumed for each slab, $z_{n}$, and associated with each such slab a set of four roots, $\zeta$, is found to exist. Two of the roots will exhibit a negative imaginary part (Im $\zeta$ negative), corresponding to an upgoing propagation component ( $+z$ direction, fig. 2$)$. Also, two of these roots will exhibit a positive imaginary part ( $\operatorname{Im} \zeta$ positive), corresponding to a downgoing propagation component (-z-direction, fig. 3). Except for the topmost slab, it is necessary to consider both upgoing and downgoing components in this analysis.

It is necessary [Johler, 1962] to equate the tangential $\vec{E}$ and $\vec{H}$ fields at each boundary, figure 2 (top and bottom of each slab), whereupon it can be concluded that a $p \times p+2$ matrix equation defines the propagation: 


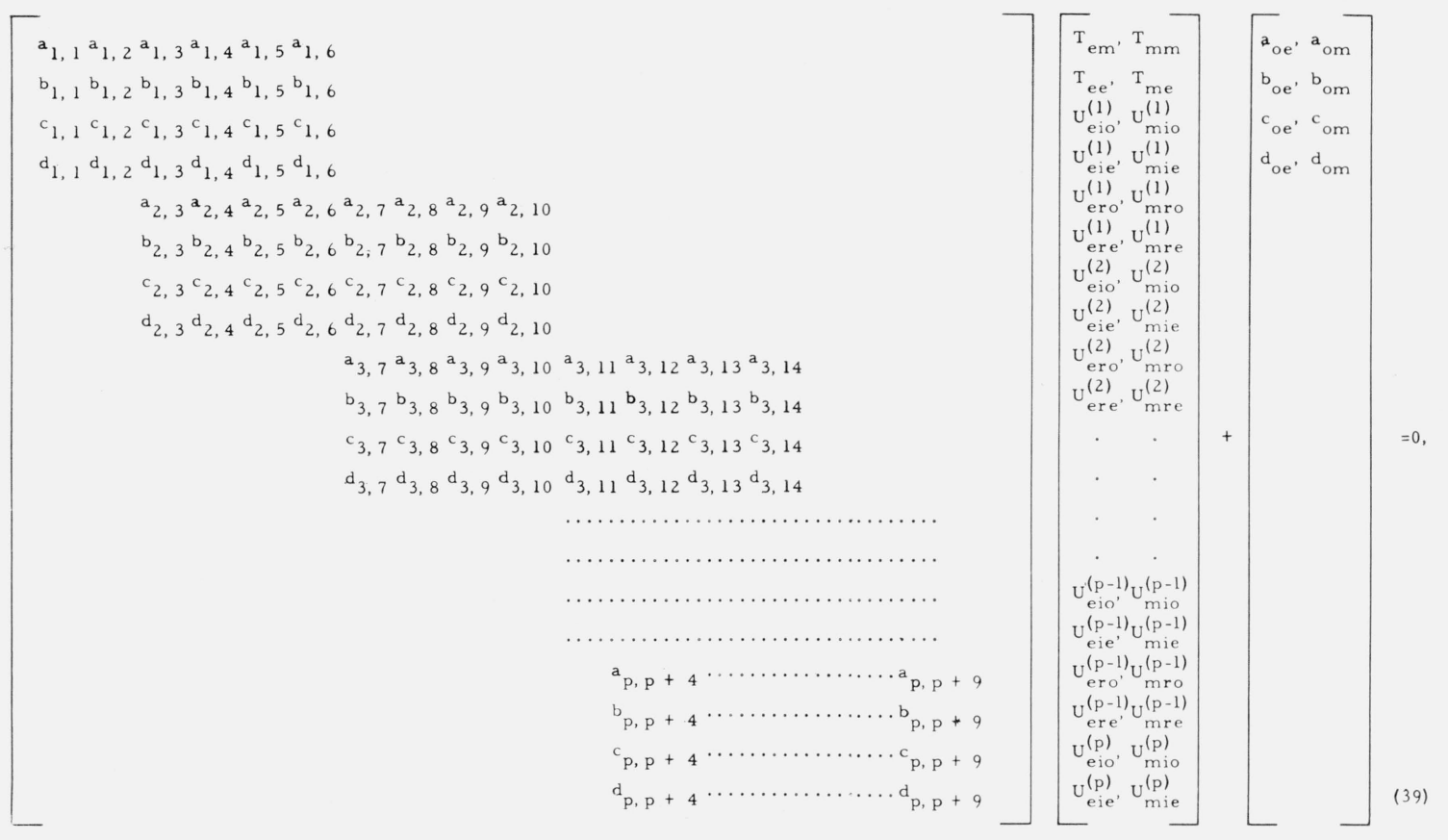

where the elements $a_{11} \ldots$. can be deduced as a consequence of these boundary conditions, provided the reflection coefficients, $T$, and the transmission coefficients, $U, T^{\prime}$ are defined,

$$
\begin{aligned}
& T_{e e}=\frac{E_{y^{\prime} r}}{E_{y^{\prime} i}} \quad T_{e e}^{\prime}=\frac{E_{y^{\prime} t}}{E_{y^{\prime} i}} \quad U_{e i o}^{(n)}=\frac{E_{y i o}^{(n)}}{E_{y^{\prime} i}} \quad U_{e r o}^{(n)}=\frac{E_{y r o}^{(n)}}{E_{y^{\prime} i}} \\
& T_{e m}=\frac{E_{x^{\prime} r}}{E_{y^{\prime} i}} \quad T_{e m}^{\prime}=\frac{E_{x^{\prime} t}}{E_{y^{\prime} i}} \quad U_{m i o}^{(n)}=\frac{E_{y i o}^{(n)}}{E_{x^{\prime} i}} \quad U_{m r o}^{(n)}=\frac{E_{y r o}^{(n)}}{E_{x^{\prime} i}} \\
& T_{m e}=\frac{E_{y^{\prime} r}}{E_{x^{\prime} i}} \quad T_{m e}^{\prime}=\frac{E_{y^{\prime} t}}{E_{x^{\prime} i}} \quad U_{e i e}^{(n)}=\frac{E_{y i e}^{(n)}}{E_{y^{\prime} i}} \quad U_{e r e}^{(n)}=\frac{E_{y r e}^{(n)}}{E_{y^{\prime} i}} \\
& T_{m m}=\frac{E_{x^{\prime} r}}{E_{x^{\prime} i}} \quad T_{m m}^{\prime \prime}==\frac{E_{x^{\prime} t}}{E_{x^{\prime} i}} \quad U_{m i e}^{(n)}=\frac{E_{y i e}^{(n)}}{E_{x^{\prime} i}} \quad U_{m r e}^{(n)}=\frac{E_{y r e}^{(n)}}{E_{x^{\prime} i}}
\end{aligned}
$$

where, figure $3, n=1,2,3 \ldots, p-1, p$, and the four $T^{\prime}$ are defined as a transmission coefficient into a topmost slab of infinite extent, $z$.

Obviously, the matrix (39) can become quite large, and the success in handling such an equation (39) is dependent upon the memory capacity of a large-scale computer (such as the IBM-7090). For example, a $104 \times 106$ complex matrix equation (39) corresponds to the upper limit of 26 slabs used on the IBM-7090 and the CDC-1604 computers [Johler and Harper, 1962a and 1962b]. The size of such slabs were approximately $0.5 \mathrm{~km}$. This was much finer detail than can ordinarily be resolved from $N(h)$ curves of the lower ionosphere. Ordinarily, only 8 to 10 slabs exhibited quite remarkable convergence of the reflection coefficients at frequencies of 10 to $100 \mathrm{kc} / \mathrm{s}$ for graphical significance in the computation.

The distinction between the four propagation components, upgoing $(i)$ and downgoing $(r)$, ordinary (o) and extraordinary (e), figure 3, becomes obscure when the ionosphere is considered to be nonuniform in the vertical $z$-direction. This is a consequence of the coupling at each boundary. Thus, the boundaries of each slab are introduced as an expression for the continuity of the tangential $\vec{E}$ and $\vec{H}$ fields and possibly the normal $\vec{H}$ at each boundary, figure 3, of the model plasmas. This is accomplished by equating the field immediately above and immediately below each boundary which, after considerable ado, results, for example, in 
a $104 \times 106$ complex matrix equation, or 26 slabs [Johler and Harper, 1962a and 1962b]. In addition to the transmission coefficients, $U$, four reflection coefficients referenced to the bottom of the lowest slab used ( $N=10, h=65$ in Houston composite model, figure $1, N=8, h=45$ in Blackout model), $T_{e e}, T_{e m}, T_{m e}, T_{m m}$ can be defined. These coefficients describe the reflection into the region below the model plasma. Thus, $T_{e e}$, used in this analysis, refers to vertical electric polarization of the incident wave and a corresponding vertical electric polarization of the reflected wave. $T_{e m}$ refers to the generation of the abnormal component (horizontal electric polarization) by the incident vertical electric wave. Similarly, $T_{m m}$ refers to vertical magnetic incident wave and vertical magnetic reflected wave, and $T_{m e}$ refers to the corresponding abnormal component (horizontal magnetic). Employing this analysis procedure, and using the vertical electric reflection coefficient, $T_{e e}$, three models were constructed as illustrated in figure 4. Model No. 1 employed the Houston Composite profile figure 2, and a distance of $1609 \mathrm{~km}$ between transmitter and receiver with typical values of the other parameters. Model No. 2 is a local ionosonde blackout model appropriate to high latitude condition in the auroral zone. Model No. 3 is the same model as No. 1, but employs a greater distance beyond the geometric-optical horizon. These models then define the propagation medium for the pulses to be described.

\section{Pulse Synthesis}

The phenomena of time separation of pulses into ground, first ionospheric reflection, etc., has been shown experimentally by Johler [1962] employing the radio-navigation-system pulse (Loran-C radio-navigation/timing system). Each such pulse can be synthesized as a superposition of three damped sinusoids as separate source-current moments.

$$
F_{s}(t)=\frac{i}{2} \exp (-\nu t)-\frac{i}{4} \exp \left(-\nu_{1} t\right)-\frac{i}{4} \exp \left(-\nu_{2} t\right)
$$

where $\nu=c_{1}+i \omega_{c}, \nu_{1}=c_{1}+i\left(\omega_{c}+2 \omega_{p}\right), \nu_{2}=c_{1}+i\left(\omega_{c}-2 \omega_{p}\right)$ and $f_{c}=\omega_{c} / 2 \pi$ frequency, characteristic. By choice of various values for $c_{1}$ and $\omega_{p}$, a variety of radio-navigation type pulses can be investigated. Methods of inserting such pulses (damped sinusoids) in the Fourier transformintegrals and evaluating such integrals numerically and analytically have been described by Johler [1962] and Johler and Walters [1959]. Thus, both the damped sine wave and the damped cosine wave source current fields can be found by evaluating the integral:

$$
\begin{aligned}
E\left(t^{\prime}, d\right)=\frac{1}{2 \pi} \int_{0}^{\infty}|E(\omega, d)| & \left\{\left[\frac{\cos \left[\omega t^{\prime}-\phi_{c}^{\prime}+\tan ^{-1} \frac{-\left(\omega_{c}+\omega\right)}{c_{1}}\right]}{\sqrt{c_{1}^{2}+\left(\omega_{c}+\omega\right)^{2}}}+\frac{\cos \left[-\omega t+\phi_{c}^{\prime}+\tan ^{-1} \frac{-\left(\omega_{c}-\omega\right)}{c_{1}}\right]}{\sqrt{c_{1}^{2}+\left(\omega_{c}-\omega\right)^{2}}}\right]\right. \\
& \left.\left.+i\left[\frac{\sin \left[\omega t^{\prime}-\phi_{c}^{\prime}+\tan ^{-1} \frac{-\left(\omega_{c}+\omega\right)}{c_{1}}\right]}{\sqrt{c_{1}^{2}+\left(\omega_{c}+\omega\right)^{2}}}\right]+\frac{\sin \left[-\omega t^{\prime}+\phi_{c}^{\prime}+\tan ^{-1} \frac{-\left(\omega_{c}-\omega\right)}{c_{1}}\right]}{\sqrt{c_{1}^{2}+\left(\omega_{c}-\omega\right)^{2}}}\right]\right\} d \omega
\end{aligned}
$$

where $\phi_{c}^{\prime}=-\phi_{c}-\pi / 2=\arg [E(\omega, d)]$ and $E(\omega, d)=E_{0}(\omega, d)$ or $E_{1}(\omega, d)$ for the groundwave or for the ionospheric wave respectively, where the field of the damped cosine source, $\operatorname{Re} F_{s}(t)$ is $\operatorname{Re}$ $E\left(t^{\prime}, d\right)$ and the field of the damped sine source, $-\operatorname{Im} F_{s}(t)$ is $-\operatorname{Im} E\left(t^{\prime}, d\right)$ or $\operatorname{Re}\left[i E\left(t^{\prime}, d\right)\right]$.

The source-current moment $\exp (-\nu t)$ is complex, since $\nu=c_{1}+i \Omega$. This is analogous to the use of complex time function $\exp (i \omega t)$ in continuous time-harmonic wave analysis. The real part is implied, 


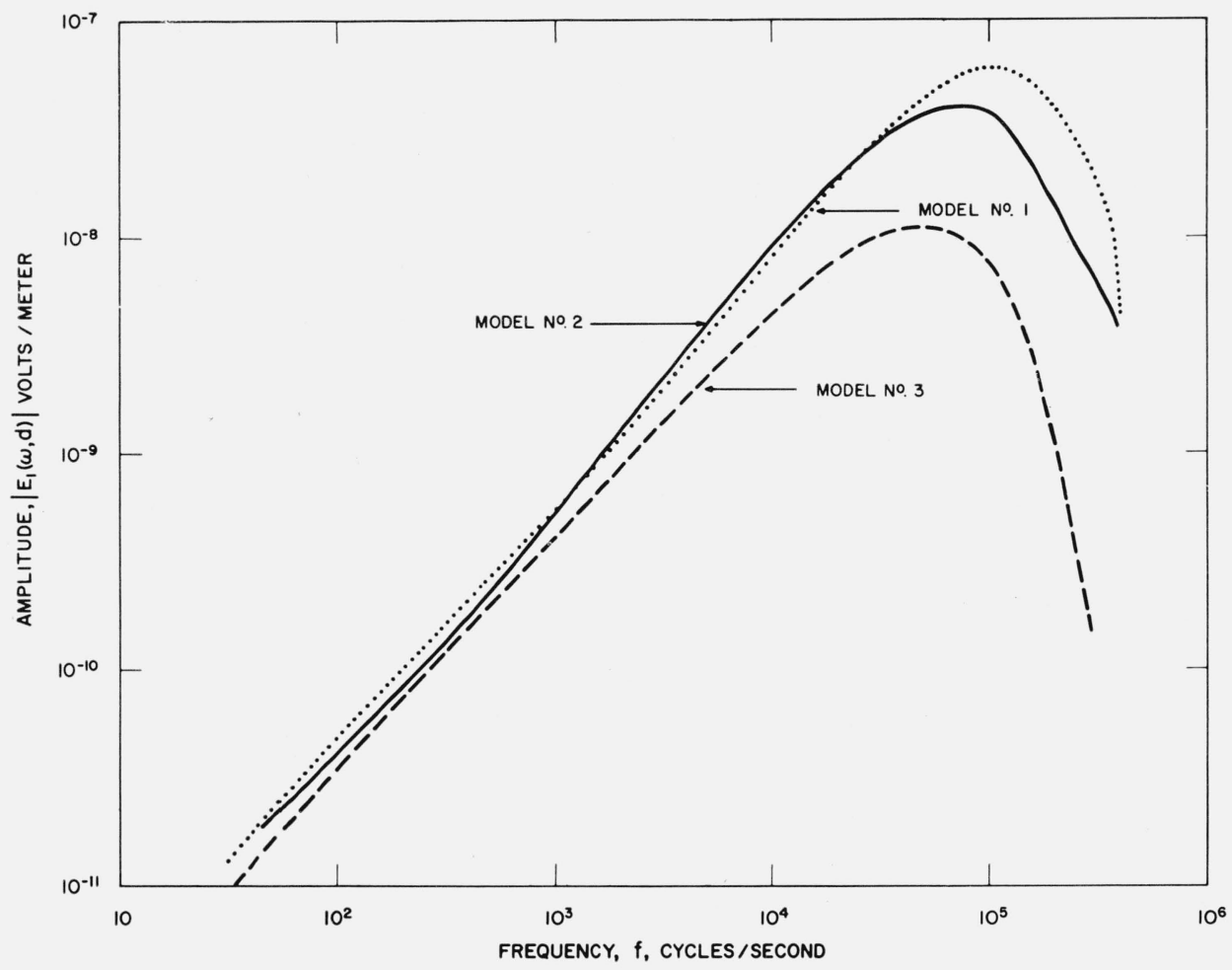

MODEL NO. I

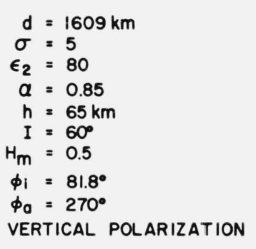

MODEL NO. 2

$d=1416 \mathrm{~km}$

$\sigma=5$

$\epsilon_{2}=80$

$\alpha=0.85$

$\mathrm{h}=45 \mathrm{~km}$

$I=60^{\circ}$

$\mathrm{H}_{\mathrm{m}}=0.5$

$\phi_{1}=83.2^{\circ}$

$\phi_{0}=270^{\circ}$

VERTICAL POLARIZATION

MODEL NO. 3

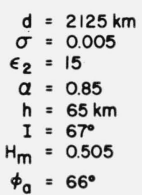

$\phi_{0}=66^{\circ}$

VERTICAL POLARIZATION

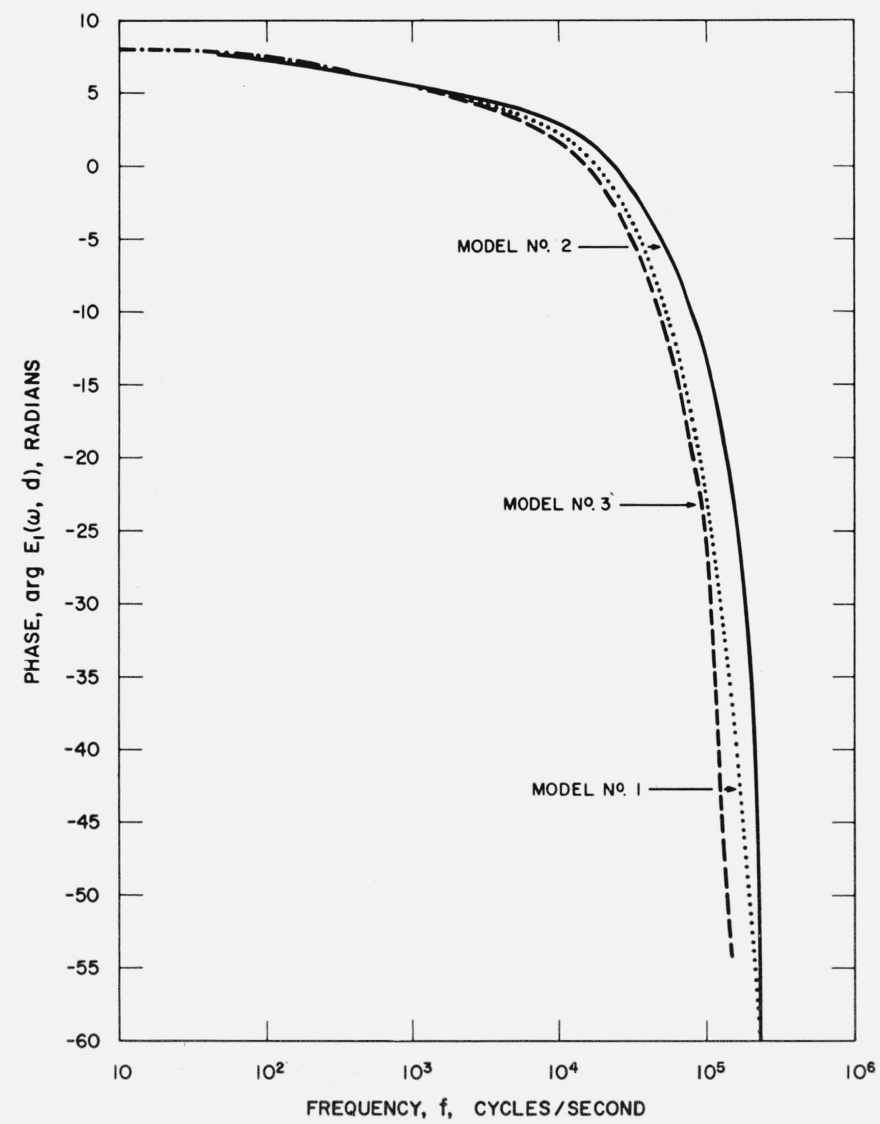

FIGURE 4. Amplitude and phase of the complex transfer function, $\mathrm{E}_{\mathrm{j}}(\omega, \mathrm{d})$, of the propagation medium for the first, $\mathrm{j}=1$, ionospheric reflection.

Three propagation medium models are illustrated: (1) The daytime-noon model for the lower geographic latitudes, distance=1609 km; (2) The local ionosonde blackout model for higher geographic latitudes (auroral zone) distance=1416 km; (3) The day time-noon model for lower geographic latitudes, distance $=2125 \mathrm{~km}$. 
$\operatorname{Re} \exp (-\nu t)=\exp \left(-c_{1} t\right) \cos (\Omega t)$

which is a damped sinusoid. The corresponding field, Re $E\left(t^{\prime}, d\right)$, is therefore implied in integral (42) as the oscillogram of the propagated pulse. The imaginary part of the integral, hence, corresponds to a source-dipole current moment, $\exp \left(-c_{1} t\right) \sin (\Omega t)$. Thus, in effect, two pulses are propagated simultaneously by the complex form of the source. This defines a source envelope, $\left|F_{s}(t)\right|$, which for the case of sinusoids has a clear physical interpretation, (both for the field, $\left|E\left(t^{\prime}, d\right)\right|$, and the source, $\left.\left|F_{s}(t)\right|\right)$ as the detected pulse envelope, corresponding to the results of an ideal detection process in a pulse receiver. Thus, the propagation of a third-type pulse wave or envelope wave is described by the integral (42). The form or shape of the envelope wave is a precise description of the dispersion of the pulse, and methods of tagging a point-in-time on the pulse to be described below employ the quantity, $\left|E\left(t^{\prime}, d\right)\right|$. It is of interest to note that the quantity, $\left|E\left(t^{\prime}, d\right)\right|$, and $\operatorname{Re} E\left(t^{\prime}, d\right)$ would be distinct even if the source, $F_{s}(t)$, were real. In other words, an envelope would be synthesized for the pulse by the integral (42) automatically.

\section{Tagging a Point-in-Time on the Pulse}

The measurement of the propagation time of a pulse or signal requires a method for tagging a point-in-time on the leading edge of the pulse. Sommerfeld [1914] and Brillouin [1914] established the consistency of signal velocity with relativity by defining such a point as the "time at which the signal takes on appreciable amplitude." Such a point-in-time while sufficient to prove the consistency of electromagnetic theory with the relativity principle, i.e., to prove that the signal always arrived at such times that the velocity of propagation is less than the speed of light, is inadequate for modern radio navigation and timing systems. Consequently, numerous precise experimental methods for defining and, indeed, tagging a point-intime on a radio pulse have been devised by Johler [1963a], Doherty et al. [1961], and Frantz, Dean, and Frank [1957]. Although one method for tagging a point-in-time on the leading edge of a pulse can be more efficient experimentally than another, the method used mathematically is inconsequential as long as the mathematics does not lose computation precision in the tagging process. A point later than $t^{\prime}=0$ is usually tagged since the signal amplitude is usually nil at zero time locally. The point tagged is inconsequential once it is tagged. Furthermore, the behavior of such a point-in-time on a pulse as distance, conductivity of the ionosphere or ground change can be studied. Such an analysis can lead to a criterion of pulse dispersion.

The amplitude envelope minus the time derivative of the amplitude envelope method [Johler 1963a] was employed in this analysis:

$$
F\left(t^{\prime}, d\right)=C_{1}\left|E\left(t^{\prime}, d\right)\right|-C_{2} \frac{d}{d t^{\prime}}\left|E\left(t^{\prime}, d\right)\right|=0 .
$$

Thus, for given values of the constant, $C_{2} / C_{1}$ the root of the differential equation (43) was found, $t^{\prime}=T_{c}$, or the zero crossings of the function, $F\left(t^{\prime}, d\right)$. The dispersion of the pulse can be studied in detail by examining several such points, $C_{2} / C_{1}$, as a function of the distance and conductivity parameters.

\section{Propagation Analysis of the Pulse}

The earliest pulse is illustrated, figure 5, at $1609 \mathrm{~km}$ (1000 statute miles); $1416 \mathrm{~km}$ (880 statute miles); and $2125 \mathrm{~km}$ (1329 statute miles). These distances, especially the latter, were selected for checking the theory of propagation experimentally. Although the transmitters and 
receivers for propagation over the distance, $2125 \mathrm{~km}$, were located on land the intervening surface was primarily sea water; hence, a conductivity, $\sigma=5$, and a dielectric constant, $\epsilon_{2}=80$, were employed for the groundwave, and a conductivity, $\sigma=0.005$, and dielectric constant, $\epsilon_{2}=15$, were selected for the factor, $F_{1}$.

The groundwave pulses, Re $E\left(t^{\prime}, d\right)$ reach a crest in approximately 5 cycles or $52 \mu$ sec, at which point the field amplitude envelope, $\left|E\left(t^{\prime}, d\right)\right|$, is $\sim 1.9\left(10^{-10}\right) \mathrm{v} / \mathrm{m}, d=2125 \mathrm{~km}$, for the assumed dipole current moment, $I_{0} l=1$. This corresponds to a radiated power, $P_{r}$, [Johler, 1961]

$$
P_{r}=1.6\left(10^{-13}\right) \omega^{2}\left(I_{0} l\right) / z_{o} \mathrm{~W}
$$

which at $f_{c}=100 \mathrm{kc} / \mathrm{s}, z_{0} \sim 120 \pi$,

$$
P_{r}=1.7\left(10^{-4}\right) \text { w peak radiated power. }
$$

Obviously the field will increase linearly with dipole moments greater than unity or increase as to the square root of the power radiated, $P_{r}$. The groundwave pulse used with the blackout model ionospheric wave was, of course, not affected by the condition of the ionosphere by definition of the groundwave pulse. The differences between the pulses are due only to the effect of distance and, hence, a greater or less effect of conductivity and earth curvature on the pulse. The vertical lapse factor, $\alpha$, and the dielectric constant would only exhibit secondary effects; hence known approximate values for these parameters were assumed.

The next pulse to arrive at the receiver, figure 6 , after considerable ionospheric wave delay (20 to $40 \mu \mathrm{sec}$ ) is the ionospheric wave pulse. The local time scale is now transformed to $t^{\prime}=t_{1}{ }^{\prime}$, i.e., the earliest time the ionospheric pulse can arrive at the receiver over the ray, $j=1$, figure 1 , is $t_{1}{ }^{\prime}=0$. The pulse corresponding to the $2125 \mathrm{~km}$ distance reaches the crest of the envelope in approximately 5 cycles or $58 \mu \mathrm{sec}$ as compared with $52 \mu \mathrm{sec}$ for the corresponding groundwave pulse, figures 5 and 6 .

The composite pulse can be formed since the pulse observed at a distant receiver is never a pure groundwave pulse or a pure ionospheric-wave pulse. There are areas of overlap [Johler, 1962]. Writing the groundwave plus the ionospheric-wave pulse,

$$
\operatorname{Re} E\left(t^{\prime}, d\right)=\operatorname{Re}\left[E_{0}\left(t^{\prime}, d\right)+E_{1}\left(t^{\prime}, d\right)\right] \text {, }
$$

or using the same time scale, $t^{\prime}$, the pulse appears as illustrated in figure 7 . The amplitude envelope $\left|E\left(t^{\prime}, d\right)\right|$ is also shown. The ionospheric-wave delay is shown. Note the short ionospheric-wave 'delay $(23.73 \mu \mathrm{sec})$ for the blackout model. This is a consequence of the lowering of the ionosphere, figure 2.

The amplitude envelope minus the time derivative of the amplitude envelope function, $F\left(t^{\prime}, d\right)$, (34) is illustrated for the three pulses for the groundwave pulse, figure 8, for the ionospheric-wave pulse, figure 9 , and for the composite pulse, figure 10 . The zero crossings were determined numerically for these pulses and the various points, $C_{2} / C_{1}$, tagged with precision are tabulated, tables 1, 2, 3, for the three models. The physical reality of the groundwave is obvious from a comparison of the pure groundwave tagged points-in-time 10.06 to $39.07 \mu$ sec, table 1 for example, with the first tagged point on the composite pulse. Since these columns are identical there is no ionospheric wave contamination. However, when the pure ionosphericwave pulse column is compared with the second tagged point-in-time on the composite wave, the contamination of the ionospheric-wave pulse by the ground-wave pulse can be noticed. The amount of signal delay for each tagged point-in-time on the ground and ionospheric-wave pulses is shown as a variable in the difference column as result of a change in the pulse dispersion.

Table 2 corresponds to a high-geographic-latitude model which is characterized by the local ionosonde blackout during which ionization extends to lower levels. The first tagged point-in-time becomes contaminated quite early on the leading edge of the composite pulse with the ionospheric-wave pulse. Thus, the point, $24.57 \mu \mathrm{sec}$ includes some of the ionospheric wave pulse. It is necessary to back up to $16.19 \mu$ sec to obtain a pure ground wave. It should 

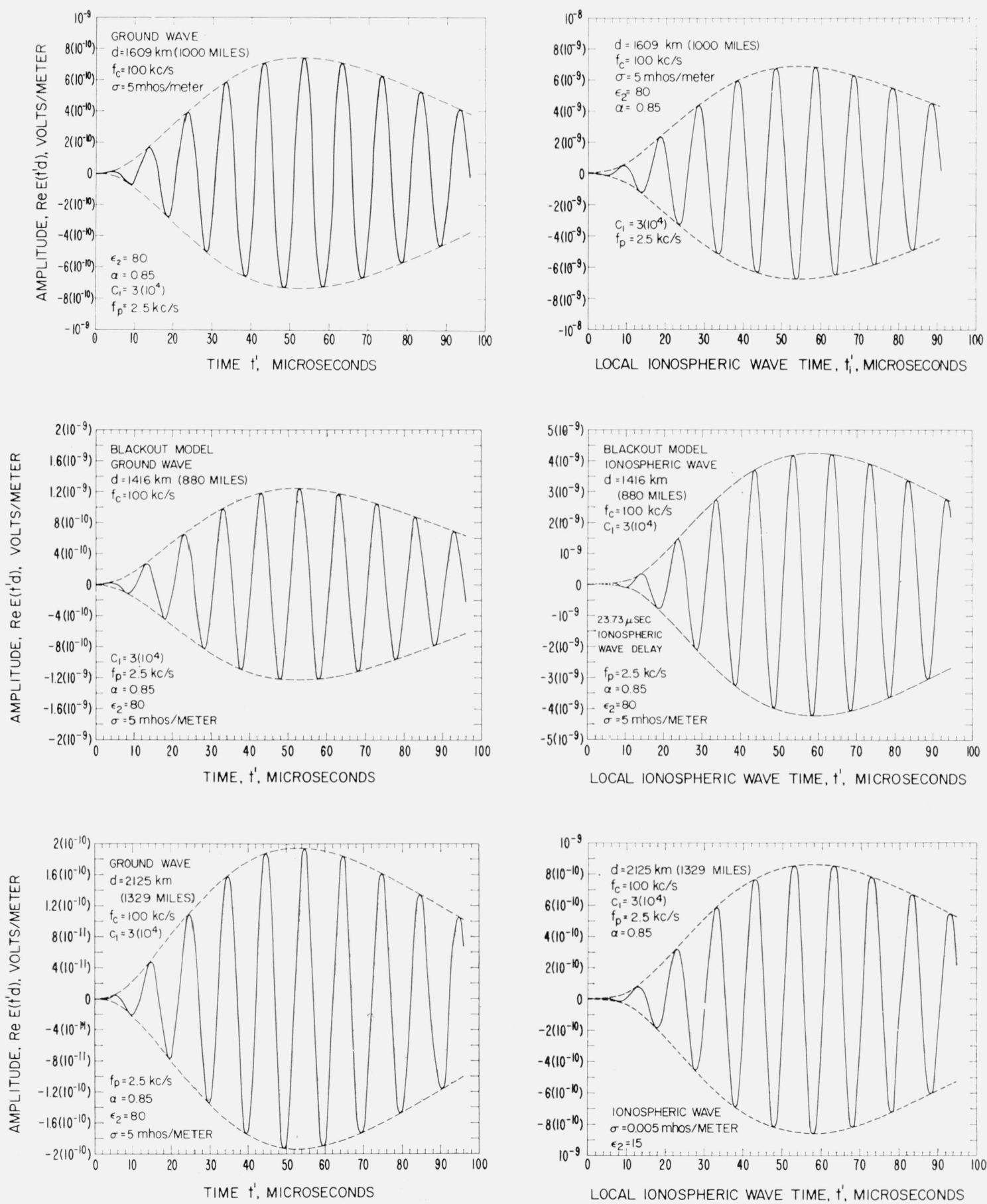

Figure 5. The ground-wave pulse, $\operatorname{Re} \mathrm{E}\left(\mathrm{t}^{\prime}, \mathrm{d}\right)$, $(\mathrm{j}=0)$, at local groundwave time, $\mathrm{t}^{\prime}=\mathrm{t}-\eta_{1} \mathrm{~d} / \mathrm{c}$, and the corresponding amplitude envelope, $\left|\mathrm{E}_{\mathrm{j}}\left(\mathrm{t}^{\prime}, \mathrm{d}\right)\right|$, illustrating a pulse signal propagated around a finitely conducting terrestrial sphere in the absence of the finitely conducting concentric plasma (ionosphere).

Figure 6. The ionospheric-wave pulse, $\operatorname{Re} \mathrm{E}_{\mathrm{j}}\left(\mathrm{t}^{\prime}{ }_{\mathrm{j}}, \mathrm{d}\right)$ $(\mathrm{j}=1)$ at local ionospheric-wave time, $\mathrm{t}^{\prime}{ }_{\mathrm{j}}$, and the corresponding amplitude envelope, $\left|\mathrm{E}_{\mathbf{j}}\left(\mathrm{t}_{\mathrm{j}}^{\prime}, \mathrm{d}\right)\right|$, illustrating a pulse signal propagated around the terrestrial sphere by reflection at the concentric plasma (ionosphere, for Models 1, 2, 3). 

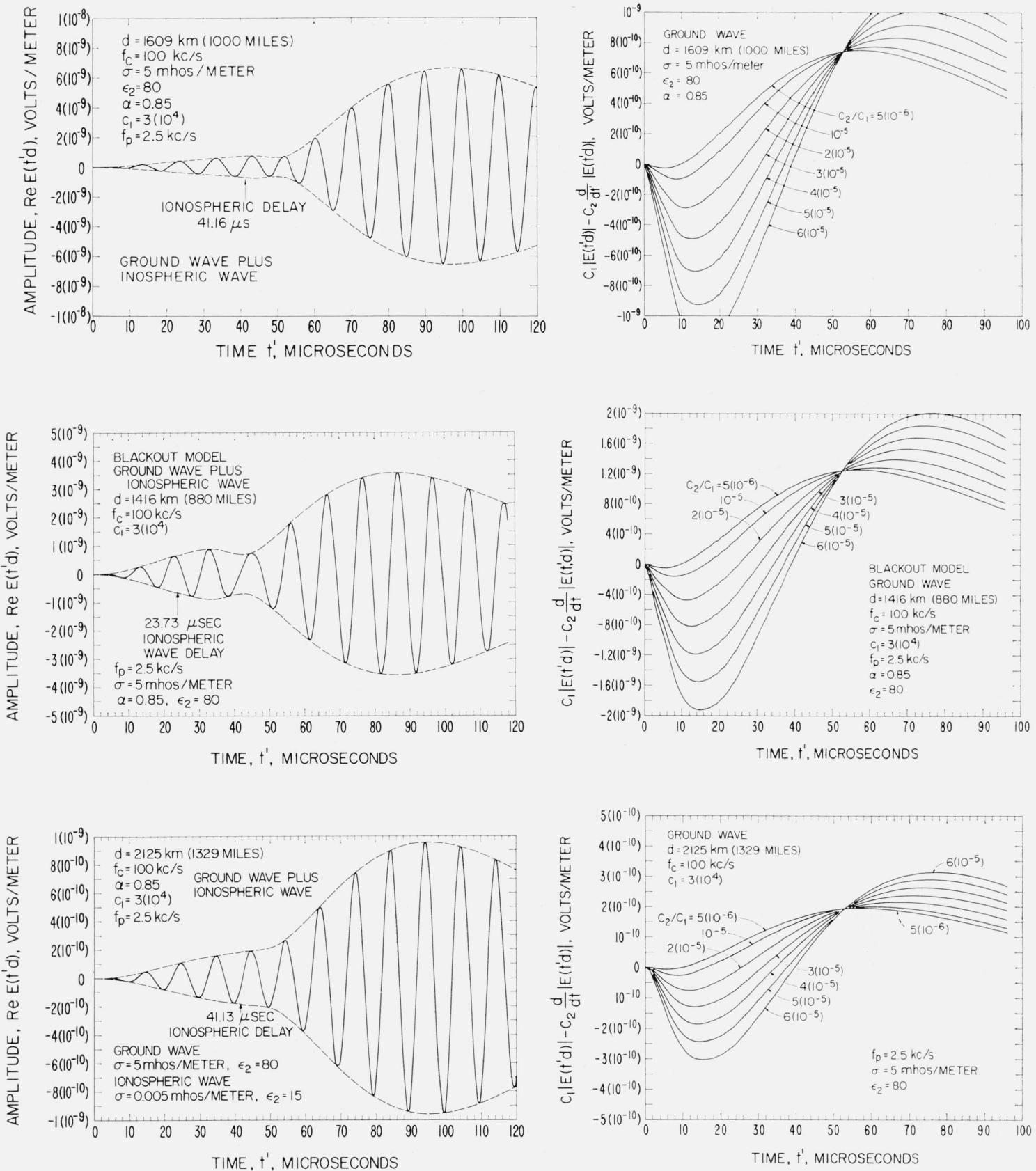

Figure 7. The composite pulse, ground-wave plus ionospheric-wave, $\operatorname{Re} \mathrm{E}\left(\mathrm{t}^{\prime}, \mathrm{d}\right)=\operatorname{Re}\left[\mathrm{E}_{0}\left(\mathrm{t}^{\prime}, \mathrm{d}\right)+\mathrm{E}_{1}\right.$ $\left.\left(\mathrm{t}^{\prime} \mathrm{d}\right)\right]$ and the corresponding amplitude envelope $\left|\mathrm{E}\left(\mathrm{t}^{\prime}, \mathrm{d}\right)\right|$; illustrating the combining, after considerable delay, of the ionospheric pulse with groundwave pulse for Models 1,2, 3 .

FIgure 8. The amplitude envelope minus the time derivative of the amplitude envelope, illustrating $a$ theoretical procedure for tagging a point-in-time on the leading edge of a ground-wave pulse, $\mathrm{d}=1609$, $1416,2125 \mathrm{~km}$. 

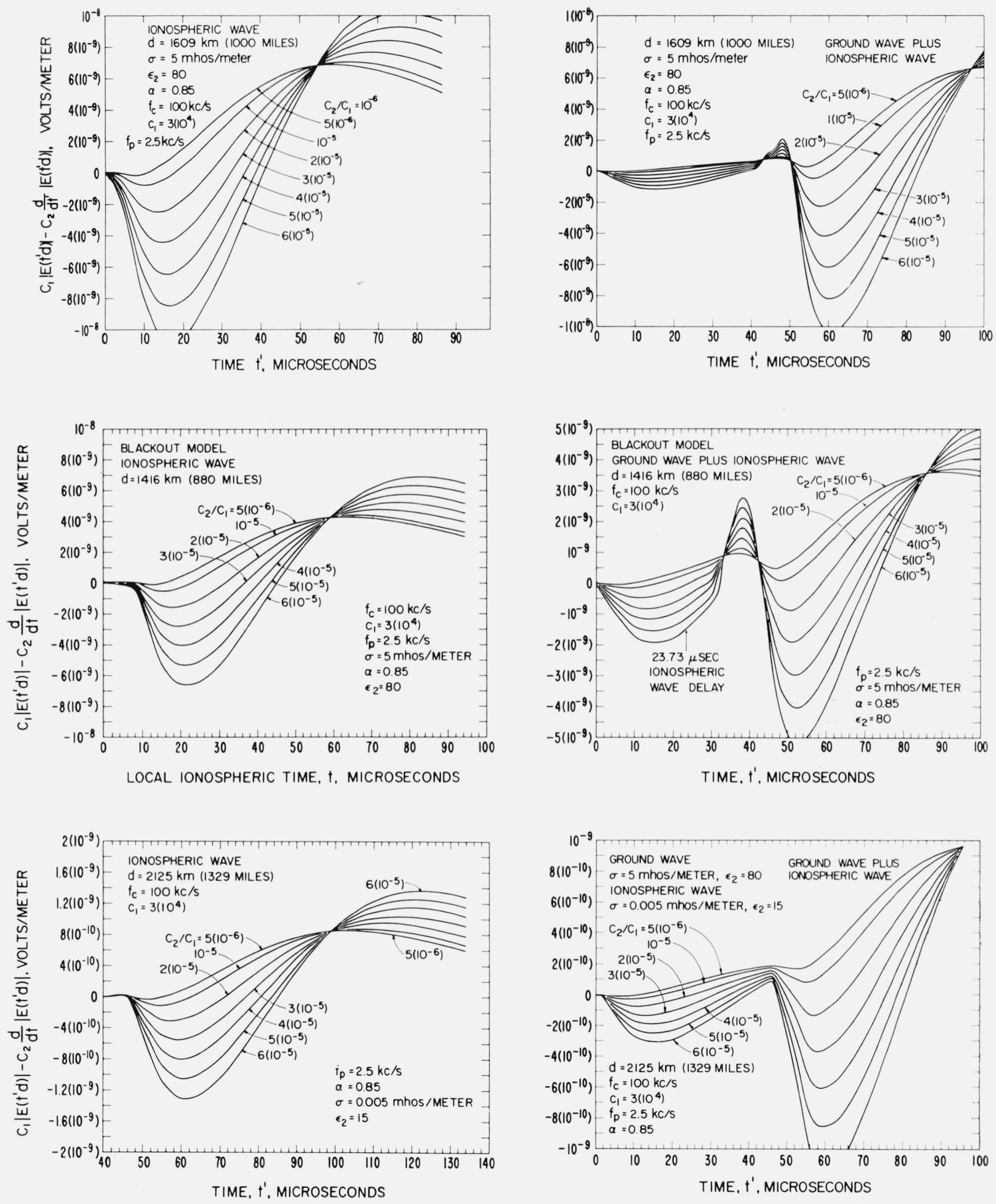

Figure 9. The amplitude envelope minus the time derivative of the amplitude envelope, illustrating a theoretical procedure for tagging a point-in-time on the leading edge of an ionospheric-wave pulse; Models 1,2, 3.

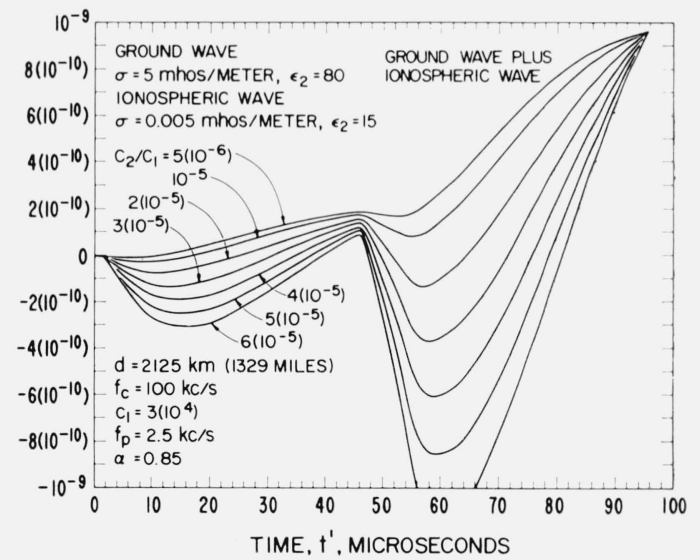

Figure 10. The amplitude envelope minus the time derivative of the amplitude envelope, illustrating $a$ theoretical procedure for tagging points-in-time on the composite ground and ionospheric-wave pulses and precisely defining an ionospheric-wave signal delay time, $\mathrm{t}_{\mathrm{c}}$, Models 1, 2, 3 . 
TABLE 1. Model 1: low-latitude, daytime-noon ionosphere

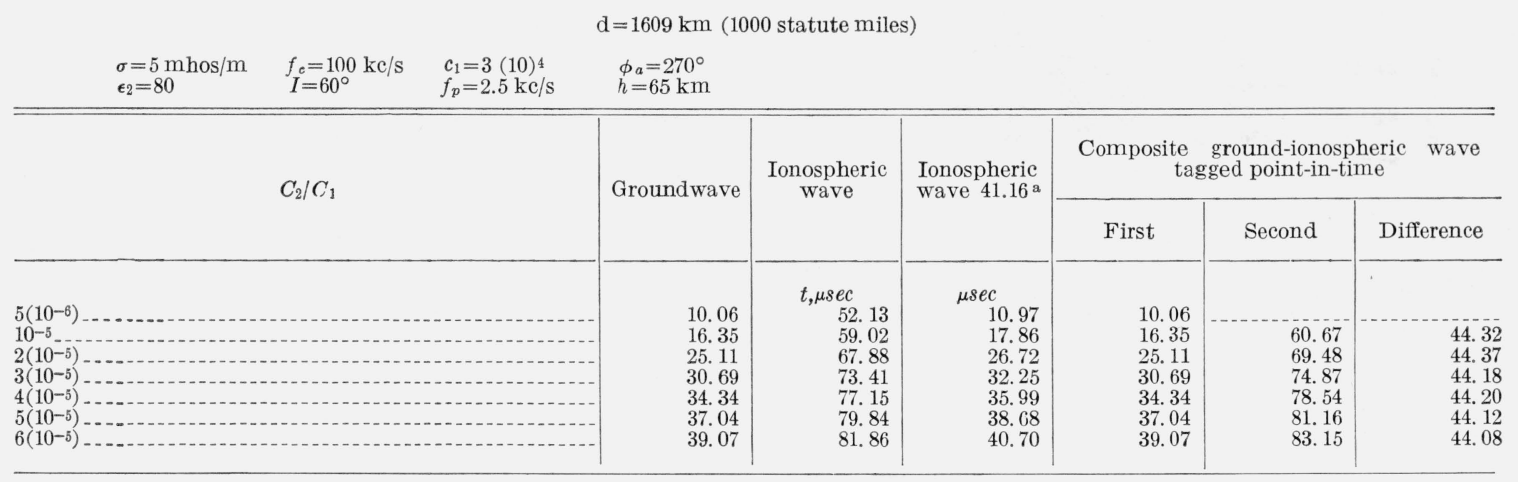

a Ionospheric wave delay relative to the groundwave-41.16 $\mu \mathrm{sec}$; continuous wave:

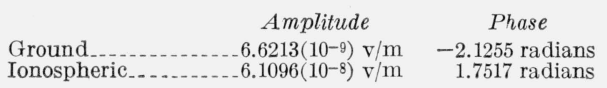

ABLE 2. Model 2: high-latitude disturbed ionosphere

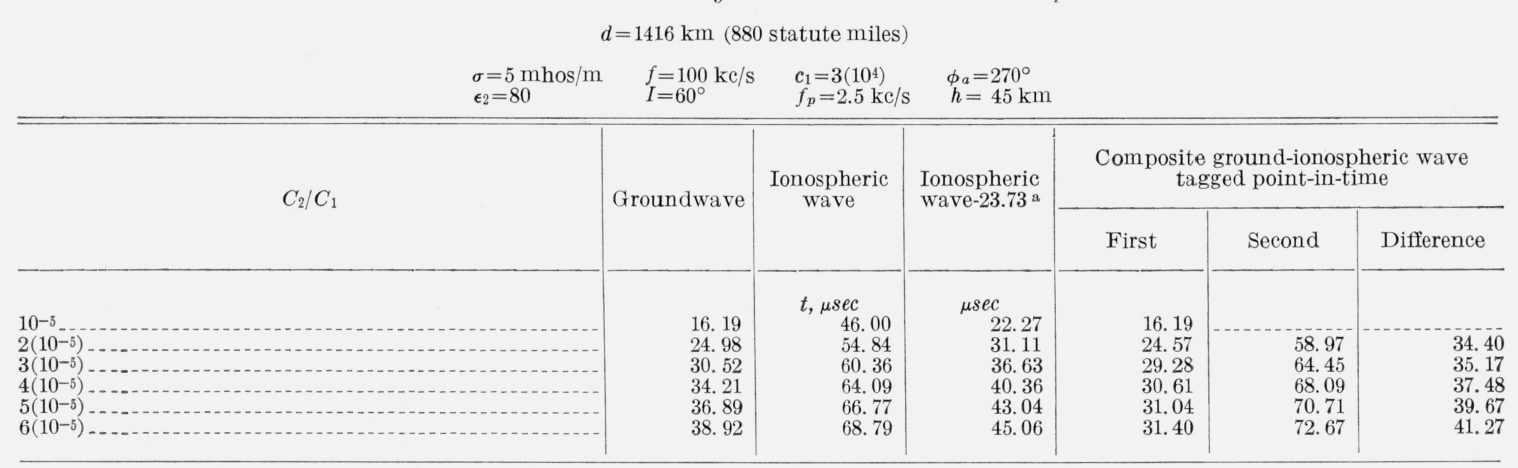

a Ionospheric wave delay relative to the groundwave $-23.73 \mu$ sec.; continuous wave:

\begin{tabular}{|c|c|c|}
\hline & Amplitude & Phase \\
\hline $\begin{array}{l}\text { Ground } \\
\text { Ionosphere }\end{array}$ & $\begin{array}{r}-1.1060\left(10^{-8}\right) \mathrm{v} / \mathrm{m} \\
3.7966\left(10^{-8}\right) \mathrm{v} / \mathrm{m}\end{array}$ & - 1.8410 radians \\
\hline
\end{tabular}

be noted that the composite wave after the arrival of the ionospheric wave contains some ground wave. This contamination with ground wave has been observed experimentally by Doherty [1963] in the Aleutian Islands and in the North Atlantic at distances between 600 and 900 statute miles employing the Loran-C radio navigation/timing system. Since the ionosphericwave delay is, in general, less in the arctic regions or northern latitudes, a reflection process at a lower altitude is indicated. The consequence of a low-level ionosphere reflection as far as the automatic equipment is concerned is illustrated by figure 10 . Note the tagged point for the Blackout Model $\left(10^{-5}\right)$, for example. The null-seeking devices which search for the zero crossing of the amplitude envelope minus the time derivative of the amplitude envelope function would never find such a point on the ionospheric pulse unless the operator reset $C_{2} / C_{1}$ to $2\left(10^{-5}\right)$. Indeed, the equipment would, if an automatic search were permitted, lock onto the ground-wave near $t^{\prime}=16 \mu$ sec during such a blackout.

Table 3 illustrates the low-geographic latitude quiescent ionosphere at great distance of $2125 \mathrm{~km}$ which is beyond the geometric-optical horizon, figure 1. Here the amount of groundwave contamination of the second point tagged on the composite pulse becomes smaller as a result of the high attenuation of the groundwave pulse, if a point is selected quite high on the pulse, $C_{2} / C_{1}=6\left(10^{-5}\right)$ or $t^{\prime}=80.82 \mu \mathrm{sec}$. 

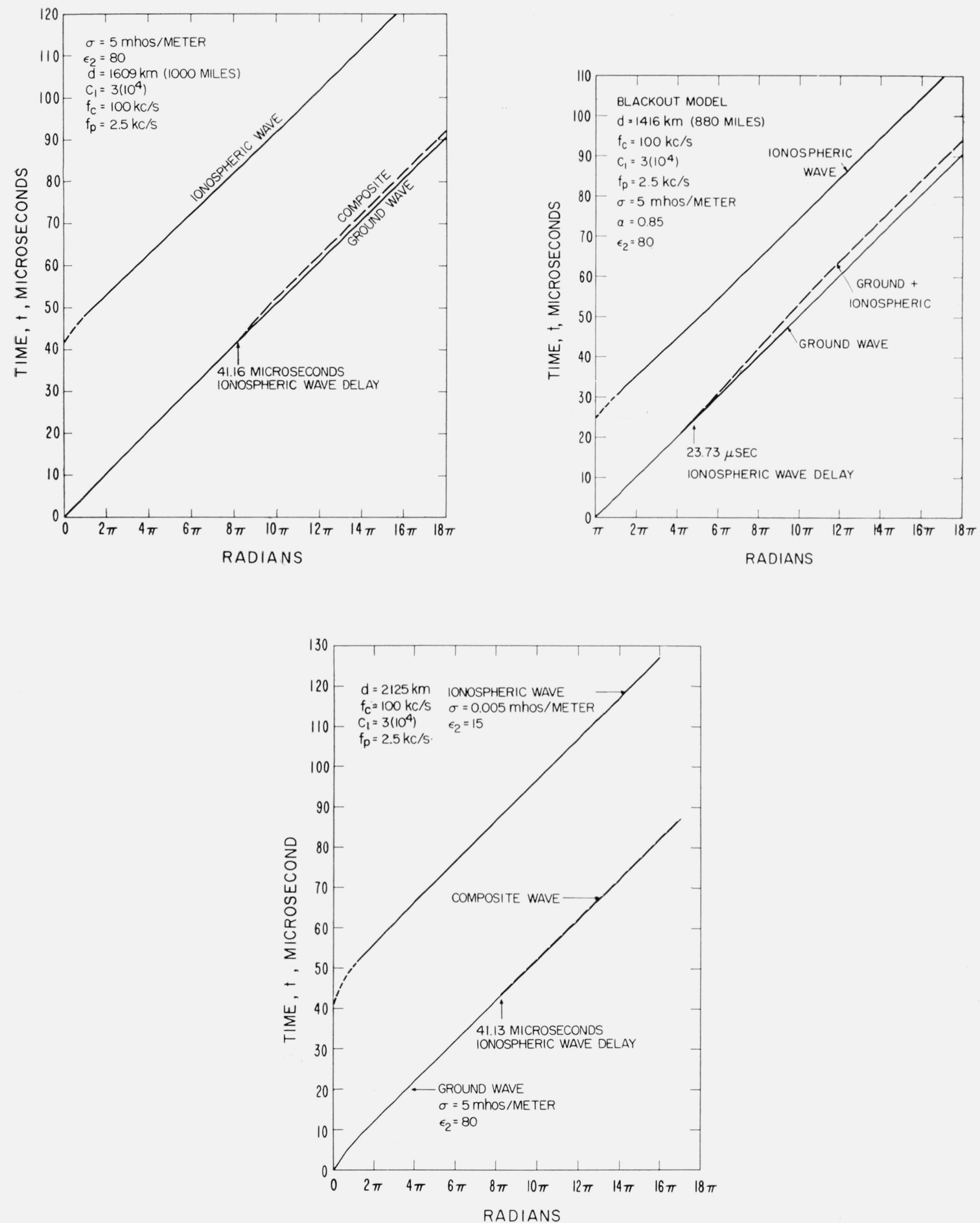

FIGURE 11. The zero crossings of the cycles on the ionospheric-wave, ground-wave, and composite pulse, illustrating cycle distortion or apparent cycle time shift on the composite pulse, Models 1, 2, 3. 
TABLE 3. Model 3; low-latitude, daytime-noon ionosphere

$d=2125 \mathrm{~km}$ (1321 statute miles)

\begin{tabular}{l}
$\begin{array}{c}f_{c}=100 \mathrm{kc} / \mathrm{s} \\
\text { Ground wave } \\
\text { Ionospheric wave }\end{array}$ \\
\hline
\end{tabular}

a Ionospheric wave delay relative to the groundwave- $41.13 ; \mu$ sec continuous wave:

\begin{tabular}{|c|c|c|}
\hline & Amplitude & Phase \\
\hline $\begin{array}{l}\text { Ground } \\
\text { Ionospheric.... }\end{array}$ & $\begin{array}{l}1.7311\left(10^{-9}\right) \mathrm{v} / \mathrm{m} \\
7.5309\left(10^{-9}\right) \mathrm{v} / \mathrm{m}\end{array}$ & $\begin{array}{l}-2.8871 \text { radians } \\
-1.1389 \text { radians }\end{array}$ \\
\hline
\end{tabular}

The corresponding time-harmonic wave solution for the ground and ionospheric wave is shown at the bottom of each table (1,2, and 3). The phase lag of the groundwave on table 1 in microseconds is $[|-2.1255| / 6.28318] 10=3.382 \mu \mathrm{sec}$ for the groundwave to which $10 \mu$ sec or $2 \pi$ radians can be added, getting $13.382 ; 23.382 \ldots \mu$ sec. This phase is ambiguous by $\pm 2 \pi$ without the pulse detection procedure outlined in this paper, i.e., without a precise method for tagging a pulse in the time domain. The phase of the continuous time-harmonic wave becomes even more obscure when applied to the composite ground and ionospheric wave. Finally the zero crossings of the individual cycles under the envelope of the pulse are illustrated, figure 11 . The curves are approximately straight lines with a $45^{\circ}$ slope. Indeed, this would be the case without cycle distortion. However, the composite wave exhibits some cycle distortion in the vicinity of overlap of the two pulses. Also, at early times on the pulse, the first cycle is distorted appreciably as shown in the $2125 \mathrm{~km}$ example, for both the ground and the ionospheric wave pulses.

\section{Conclusions}

The methods which employ the direct analytic or numerical procedures for the evaluation of the Fourier transform-integral for the groundwave pulse can be applied to ionospheric wave pulses. The form or shape of the composite pulse compares very well with those observed in practice. The phenomenon of time separation of pulses observed experimentally has been demonstrated mathematically. The complete detailed analysis of pulse dispersion, signal velocity, and ionospheric wave pulse or groundwave pulse timing requires precise methods for tagging a point-in-time on the pulse-both mathematical and experimental. One such mathematical method has been successfully applied in this analysis.

\section{References}

Berry, L. A. (1962), private communication.

Booker, H. G. (1939), Propagation of wave packets incident obliquely upon a stratified doubly refracting ionosphere, Phil. Trans. Roy. Soc. London, Ser. A, 227, 411-451.

Brillouin, L. (1914), Über die Fortpflanzung des Lichtes in dispergierenden Medien, Ann. Physik, Vierte Folge, Band 44, No. 10.

Doherty, R. H. (1963), private communication.

Doherty, R. H., G. Hefley, and R. F. Linfield (Nov. 1961), Timing potentials of Loran-C, Proc. IRE 49, No. 11 1659-1673. 
Frantz, Wilbert P., Walter N. Dean, and Robert L. Frank (1957), A precision multi-purpose radio navigation system, IRE Convention Record, Pt. I, Characteristics and Applications.

Johler, J. R. (Sept.-Oct. 1961), On the analysis of LF ionospheric radio propagation phenomena, J. Research 65D (Radio Prop.) No. 5, 507-529.

Johler, J. R. (Apr. 1962), Propagation of the low-frequency radio signal, Proc. IRE 50, No. 4, $404-427$.

Johler, J. R. (1963a), On radio wave reflections at a continuously stratified plasma with collisions proportional to energy and arbitrary magnetic induction, Proceedings of the International Conference on the Ionosphere, London 1962, pp. 436-445 (The Institute of Physics and the Physical Society, 1 Lowther Gardens, Prince Consort Road, London, S.W. 7).

Johler, J. R. (1963b), On the propagation time of a radio pulse, IEEE Trans. Ant. Prop. (Nov. 1963).

Johler, J. R., and John D. Harper, Jr. (Jan.-Feb. 1962a), Reffection and transmission of radio waves at a continuously stratified plasma with arbitrary magnetic induction, J. Research 66D (Radio Prop.), No. 1, 81-99.

Johler, J. R., and John D. Harper, Jr. (1962b), On plasma collision frequencies proportional to energy in the radio wave reflection and transmission process, NBS Tech. Note No. 164.

Johler, J. R., and L. C. Walters (Jan. 1959), Propagation of a groundwave pulse around a finitely conducting spherical earth from a damped sinusoidal source current, IRE Trans. Ant. Prop. AP-y, No. 1, 1-10.

Johler, J. R., L. C. Walters, and C. M. Lilley (1959), Low- and very low-radio-frequency tables of groundwave parameters for the spherical earth theory: The roots of Riccati's differential equation, NBS Tech. Note No. 7, PB-151366.

Keilson, J., and R. V. Row (Oct. 1959), Transfer of transient electromagnetic surface waves into a lossy medium, J. Appl. Phys. 30, No. 10, 1595-1598.

Novikov, V. V. (1960), Propagation of a radio pulse over the surface of a plane homogeneous earth, Vestnik Leningradskogo Universiteta Seria Fiziki i Khimii, Nr. 10, p. 16 (Issue 2).

Pekeris, C. L., and Z. Alterman (Nov. 1957), Radiation resulting from an impulsive current in a vertical antenna placed on a dielectric ground, J. Appl. Phys. 28, No. 11, 1317-1323.

Sommerfeld, A. (1914), Über die Fortpflanzung des Lichtes in dispergierenden Medien, Ann. Physik, Vierte Folge, Band 44, No. 10.

Wait, J. R. (Jan. 1956), Transient fields of a vertical dipole over a homogeneous curved ground, Can. J. Phys. 34, 27-35.

Wait, J. R. (Apr. 1957), The transient behavior of the electromagnetic groundwave on a spherical earth, IRE Trans. Ant. Prop. AP-5, No. 2, 198-202.

Wait, J. R., and C. Froese (Mar. 1955), Reflection of a transient electromagnetic wave at a conducting surface, J. Geophys. Res. 60, No. 1, 97-103.

Wait, J. R., and Alyce M. Conda (Oct. 1958), Pattern of an antenna on a curved lossy surface, IRE Trans. Ant. Prop. AP-6, 348-359.

Wait, J. R., and Alyce M. Conda (1959), On the diffraction of electromagnetic pulses by curved conducting surfaces, Can. J. Phys. 37, 1384-1396.

(Paper 67D5-281) 